\title{
Small-world networks of Kuramoto oscillators
}

\author{
Georgi S. Medvedev*
}

November 25, 2013

\begin{abstract}
The Kuramoto model of coupled phase oscillators on small-world (SW) graphs is analyzed in this work. When the number of oscillators in the network goes to infinity, the model acquires a family of steady state solutions of degree $q$, called $q$-twisted states. We show that this class of solutions plays an important role in the formation of spatial patterns in the Kuramoto model on SW graphs. In particular, the analysis of $q$-twisted states elucidates the role of long-range random connections in shaping the attractors in this model.

We develop two complementary approaches for studying $q$-twisted states in the coupled oscillator model on SW graphs: linear stability analysis and numerical continuation. The former approach shows that long-range random connections in the SW graphs promote synchronization and yields the estimate of the synchronization rate as a function of the SW randomization parameter. The continuation shows that the increase of the long-range connections results in patterns consisting of one or several plateaus separated by sharp interfaces.
\end{abstract}

These results elucidate the pattern formation mechanisms in nonlocally coupled dynamical systems on random graphs.

\section{Introduction}

Coupled dynamical systems on graphs model the evolution of complex networks of diverse nature. Examples include neuronal networks in biology [6]; Josephson junctions and coupled lasers in physics [14, 24]; communication, sensory, and power networks in technology [8], to name a few. In these models, each node of the graph supports a local dynamical system, whose dynamics is typically well understood. Local systems at the adjacent nodes interact with each other, thus, producing collective dynamics of the coupled system. The spatial structure of the network may vary from regular as in lattice dynamical systems to completely random. Thus, it is not surprising that dynamical systems on graphs exhibit a wide range of spatio-temporal dynamics.

The main challenge in studies of dynamical networks has been relating the structure of the graph of the network to its dynamics. In view of the enormous complexity of this class of systems, our understanding of the link between the structure and dynamics has been guided by the analysis of certain successful models

${ }^{*}$ Department of Mathematics, Drexel University, 3141 Chestnut Street, Philadelphia, PA 19104, medvedev@drexel . edu 

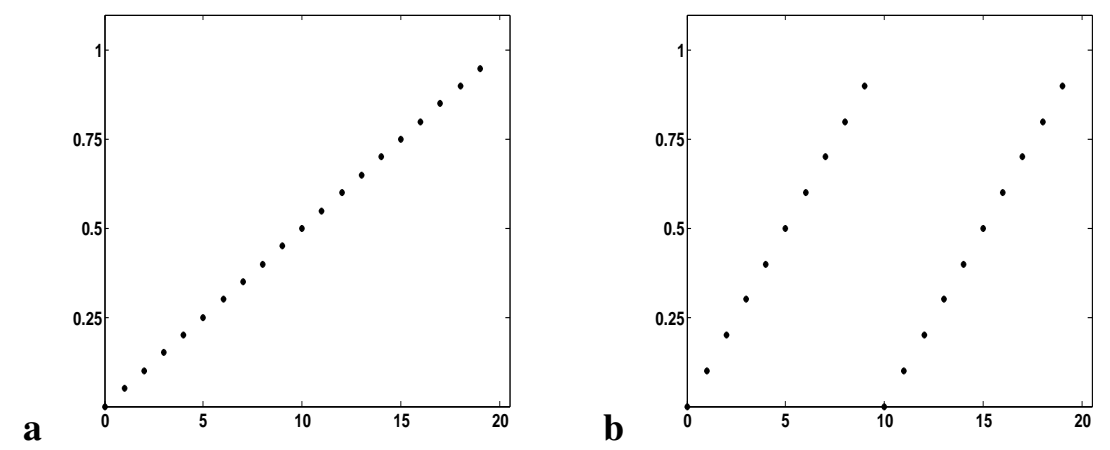

Figure 1: Plots of 1- and 2-twisted states, steady state solutions of the Kuramoto model of 20 coupled oscillators, are shown in $\mathbf{a}$ and $\mathbf{b}$ respectively.

illuminating various aspects of network dynamics. In this respect the Kuramoto model of coupled oscillators stands out as one of the most influential in the field [13]. Derived as the phase description of large ensembles of limit cycle oscillators, the Kuramoto model has been used by physicists and biologists to gain insights into many important effects in large networks such as synchronization, multistability, phase transitions, wave propagation, and spatio-temporal chaos, to name a few [11]. Recent experiments with the Kuramoto model with nonlocal coupling led to the discovery of chimera states, spectacular patterns combining coherent and irregular turbulence-like behavior [12].

The Kuramoto model describes dynamics of coupled phase oscillators on a graph. Thus, before we introduce the model, we need to review some graph-theoretic notation. Let $G=\langle V(G), E(G)\rangle$ be an undirected graph, where $V(G)$ stands for the set of nodes of $G$, and the edge set $E(G) \subset V(G)^{2}$ contains the unordered pairs of connected nodes. Furthermore, $G$ is called a simple graph, if it does not contain loops or multiple edges.

Let $\left\{G_{n}, n \in \mathbb{N}\right\}$ be a sequence of simple graphs. If $\left|E\left(G_{n}\right)\right|=O\left(\left|V\left(G_{n}\right)\right|^{2}\right)$ then graphs $G_{n}, n \gg 1$, are called dense.

The following graph sequence will appear frequently throughout this paper. For sufficiently large $n \in \mathbb{N}$ and $k \in \mathbb{N}$ such that $2 k<n$, we define a sequence of $k$-nearest-neighbor $(k-\mathrm{NN})$ graphs $\left\{C_{n, k}\right\}$ as follows

$$
V\left(C_{n, k}\right)=[n] \quad \text { and } \quad E\left(C_{n, k}\right)=\left\{(i, j) \in[n]^{2}: 0<d_{n}(i, j) \leq k\right\}
$$

where $d_{n}(i, j)=\min \{|i-j|, n-|i-j|\}$. Let $r \in(0,1 / 2)$ then $\left\{C_{n,\lfloor r n\rfloor}\right\}$ is a dense graph sequence.

We now introduce the Kuramoto model of coupled phase oscillators on $\left\{G_{n}\right\}$

$$
\frac{d}{d t} u_{n i}(t)=\omega_{i}+\frac{\sigma}{n} \sum_{j:(i, j) \in E\left(G_{n}\right)} \sin \left(2 \pi\left(u_{n j}(t)-u_{n i}(t)\right)\right), i \in[n],
$$

where $u_{n i}: \mathbb{R}^{+} \rightarrow \mathbb{S}^{1}:=\mathbb{R} / \mathbb{Z}, i \in[n]$, models the evolution of the phase oscillator $i$, and $\omega_{i}$ is its intrinsic frequency. The scaled sum on the right hand side of (1.2) describes the interactions between oscillator $i$ and the oscillators connected with it. In the classical Kuramoto model, $\sigma=1$. This is so-called attractive coupling case, because when $\sigma=1$, synchrony is stable. Recently, there has been an interest in studying 
the Kuramoto model with $\sigma=-1$, i.e., the repulsive coupling case [10]. Existence of steady state solutions clearly does not depend on the sign of $\sigma$. However, the stability of these solutions does. In this paper, we are interested in the case of dense graphs $\left\{G_{n}\right\}$, which corresponds to the nonlocal coupling in the Kuramoto model (1.1).

If all intrinsic frequencies $\omega_{i}, i \in[n]$, are equal, as we assume for the remainder of this paper, they can be eliminated by recasting $(1.1)$ in the rotating frame of coordinates. Thus, we consider

$$
\frac{d}{d t} u_{n i}(t)=\frac{\sigma}{n} \sum_{j:(i, j) \in E\left(G_{n}\right)} \sin \left(2 \pi\left(u_{n j}(t)-u_{n i}(t)\right)\right), i \in[n] .
$$

Our work was inspired by the analysis of the Kuramoto model on the sequence of $k$-NN graphs $\left\{C_{n, k}\right\}$, $k=\lfloor r n\rfloor, r \in(0,1 / 2)$ in [26]. In this paper, Wiley, Strogatz, and Girvan identified an important class of steady state solutions of degree $q(0 \leq q \leq n)$,

$$
u_{n}^{(q)}=\left(u_{n 1}^{(q)}, u_{n 2}^{(q)}, \ldots, u_{n n}^{(q)}\right), u_{n i}^{(q)}=\frac{q(i-1)}{n}+c \quad(\bmod 1), c \in[0,1), i \in[n] .
$$

These solutions are called $q$-twisted states. The 0-twisted states correspond to spatially uniform (synchronous) solutions. Steady states with $q \neq 0$ form linear patterns that wind around $\mathbb{S}^{1} q$ times (see Fig. 1 ). It was shown in [26] that the stability of $q$-twisted states for the Kuramoto model depends on the range of nonlocal coupling $r$. This provides a nice case study illustrating how the network topology is shaping the structure of the phase space of the coupled system. The follow-up study of $q$-twisted states in the Kuramoto model with repulsive coupling suggests their relevance to the chimera states and, more generally, to the coherence-incoherence transition in coupled systems [10].

Interestingly, the original motivation for the analysis in [26] was the dynamics on SW networks. SW graphs, proposed by Watts and Strogatz, interpolate between graphs with regular local connections and completely random Erdős-Rényi graphs [25]. In the classical Watts-Strogatz SW graph, one starts with the $k$-NN graph on a circle $C_{n, k}(n>2 k)$. Then each edge in $C_{n, k}$ is replaced with probability $p \in[0,1]$ to a randomly chosen one (see Fig. 2). We will refer to $p$ as the randomization parameter. If $p=1$ then $C_{n, k}$ is transformed into the Erdős-Rényi graph. For analytical convenience Newman and Watts and independently Monasson used another variant of SW connectivity, where the new random edges are added to the graph, while no edges are removed [20, 19].

Like many other random graphs, SW graphs have small average path length, but they also have large clustering coefficients inherited from the regular graphs. The combination of these two properties has been shown in many real-life networks, including neuronal networks [25, 3], the power grid of the western United States, and professional networks [25]. Thus, studying dynamical systems on SW graphs has the potential of elucidating principles governing dynamics in diverse realistic networks. A specific question posed in [26] is how adding a small number of random long range connections to otherwise regular network affects stability of the synchronous state. The synchronizing effect of long-range connections was suggested by the previous studies of related models [21, 2]. However, the rigorous understanding of this effect in SW networks was lacking.

In this paper, we study the role of the long-range random connections in shaping stationary solutions of the Kuramoto model on SW graphs. For this, we use two complementary approaches: the linear stability 

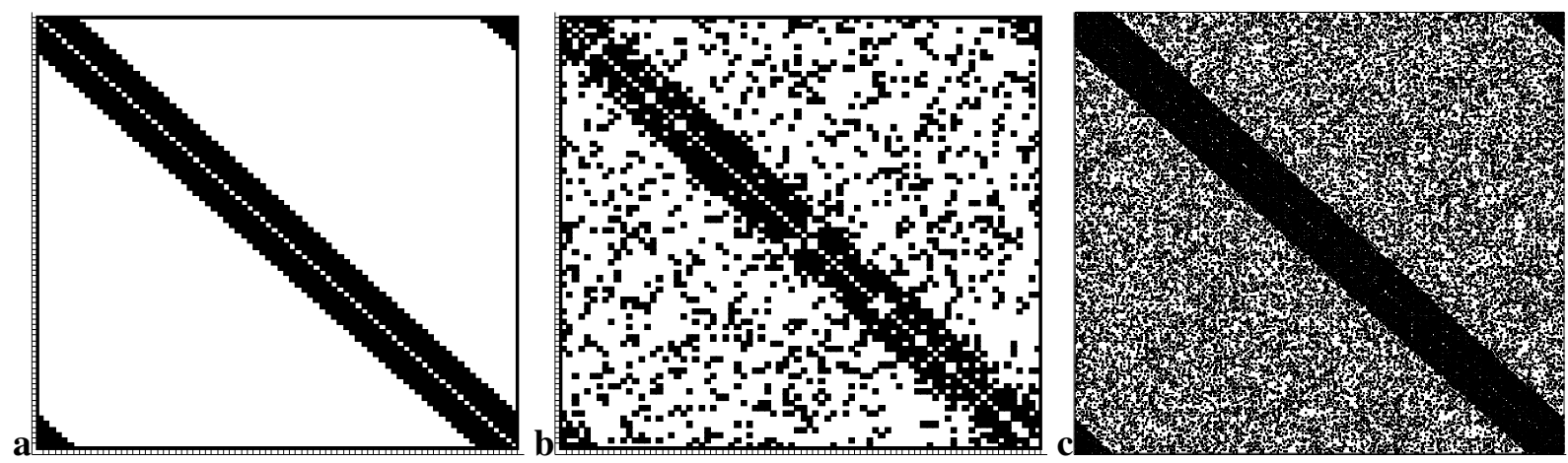

Figure 2: a) A graphical representation of the adjacency matrix of $C_{n, k}$. In this example $k=6$ and $n=80$, i.e., each of the 80 nodes on the ring is connected to 6 nearest neighbors from each side. The horizontal and vertical sides of each square are divided into intervals corresponding to the nodes of the graph. A black cell at the intersection of a given row and a column indicates a pair of connected nodes. b) The SW graph obtained by replacing each (local) edge in $C_{80,6}$ by a random long-range connection with probability $p=0.2$. For increasing values of $p$, the distribution of the black cells approaches the uniform distribution, i.e., the SW graphs become progressively more similar to the Erdős-Rényi graph. c) The SW graph obtained by replacing each edge in $C_{400,30}$ by a random long-range connection with probability $p=0.2$.

analysis of $q$-twisted states and numerical continuation. Neither of this approaches can be applied to the problem as it stands, and, therefore, some preliminary work is required. We comment on the stability analysis first. In [26], Wiley, Strogatz, and Girvan obtained a sufficient condition for stability of $q$-twisted states for the $k$-NN coupled Kuramoto model. Their analysis employed the continuum limit of the Kuramoto model. Unlike the regular $k$-NN coupling case, on SW graph when the number of oscillators $n$ is finite, $q$ twisted states are no longer solutions of the Kuramoto equation. We show that they become steady state solutions again in the appropriate probabilistic sense in the limit as $n \rightarrow \infty$. Thus, as before the continuum limit provides a natural setting for the stability analysis. Our previous work [18] shows how to derive continuum limits for the Kuramoto model on W-random graphs including the SW graphs. This allows the stability analysis of the $q$-twisted states for the SW model to be performed in analogy to the analysis of the deterministic $k$-NN networks in [26].

The stability analysis of $q$-twisted states in the continuous setting clearly shows the respective contributions of the regular local and random long-range connections of the SW network topology to the spectrum of the linearized problem. It shows that adding new long-range random connections enhances stability of the synchronous regime, while inhibiting other $q$-twisted states. Furthermore, the estimate of the leading eigenvalue of the linearized problem yields the synchronization rate as a function of the randomization parameter $p$. The synchronization rate grows monotonically with $p$. Thus, our quantitative estimates of the eigenvalues of the linearized problem show that adding new random connections in the SW topology strengthens the synchrony.

As a complementary approach aimed at elucidating the impact of long-range connections on the solutions of the Kuramoto model and on the spatial patterns that it forms, we developed a continuation method that reveals the transformations of the $q$-twisted states under the variation of the randomization parameter $p$. The major obstacle in implementing the continuation procedure was the fact that in general the right hand side of the Kuramoto model is not continuous in $p$. This is easy to understand by noting that the adjacency 
matrices of two independent copies of SW graphs, $G_{n, p_{1}}$ and $G_{n, p_{2}}$, do not have to be close when $\left|p_{2}-p_{1}\right|$ is small or even when it is equal to 0 . To overcome this problem we constructed a special family of SW graphs $G_{n, p}$, whose members depend continuously on $p$. Here, the continuity is meant in the appropriate probabilistic sense (see Section 5 for details). We call such family of SW graphs consistent. With the consistent family of SW graphs at hand, we set up a numerical continuation algorithm based on the Newton's method and computed branches of steady state solutions of the Kuramoto model on SW graphs for $p \geq 0$ by starting from $q$-twisted states for $p=0$. The numerical continuation revealed an unexpected effect. It turns out that for increasing values of $p$, the $q$-twisted states are transformed into patterns composed of $q$ plateaus separated by interfaces. We observed this effect in all random realizations of the consistent families of SW graphs that we used in our experiments. The formation of plateaus in solutions continued from $q$-twisted states is another manifestation of the synchronizing effect of the long-range connections.

This paper is organized as follows. In Section 2, we redefine SW graphs using the framework of Wrandom graphs [18] and derive the continuum limit of the Kuramoto model on SW graphs. In Section 3 , we show that in the limit as $n \rightarrow \infty, q$-twisted states become stationary solutions of the Kuramoto model on SW graphs. This is followed by the linear stability analysis of $q$-twisted states. The implications of the stability analysis for synchronization are discussed in Section 4 . The numerical continuation from the $q$-twisted states is developed in Section 5. The final Section 6 contains concluding remarks.

\section{The continuum limit of the Kuramoto model}

The stability analysis of $q$-twisted states in Section 3 uses the continuum (thermodynamic) limit of the Kuramoto equation (1.2) as $n \rightarrow \infty$. The thermodynamic limit has proved to be a useful tool for elucidating various aspects of the dynamics of large networks (see, e.g., [12, 1, 26, 10, 22]). In [17], we provided a rigorous justification of the continuum limit for the models of Kuramoto type on dense graphs, using the ideas from the theory of graph limits [15, 16, 5]. This work was further extended to cover dynamical systems on random graphs in [18]. In particular, the analysis in [18] shows how to set up and to justify the continuum limit for the Kuramoto model on the SW graphs. Before we apply the continuum limit to study the stability of $q$-twisted states, we review a few facts illuminating the basis for the limiting dynamics of (1.2) as $n \rightarrow \infty$.

As the first step toward the continuum limit, we redefine SW graphs as W-random graphs [15, 16]. The latter provide a flexible framework for constructing random graphs, which is especially convenient for studying coupled dynamical systems. Below, we review the construction of W-random graphs and use it to define SW graphs (cf. [18]).

Let $\mathcal{W}_{0}$ be a class of symmetric measurable functions on $I^{2}$ with values in $I$. Here and throughout this paper, $I$ stands for the unit interval $[0,1]$.

Definition 2.1. $[16]$ Let $W \in \mathcal{W}_{0}$ and

$$
X_{n}=\left\{x_{1}, x_{2}, x_{3}, \ldots, x_{n}\right\}
$$

be a set of distinct points from I. Define $G_{n}=\left\langle[n], E\left(G_{n}\right)\right\rangle$ such that for each $(i, j) \in[n]^{2}$

$$
\mathbb{P}\left\{(i, j) \in E\left(G_{n}\right)\right\}= \begin{cases}W\left(x_{i}, x_{j}\right), & i \neq j \\ 0, & \text { otherwise }\end{cases}
$$


where $\mathbb{P}\{\cdot\}$ stands for the probability of an event. Graph $G_{n}$ is called a $W$-random graph and denoted by $G_{n}=\mathbb{G}\left(W, X_{n}\right)$.

In the remainder of this paper, we use

$$
X_{n}=\left\{0, \frac{1}{n}, \frac{2}{n}, \ldots, \frac{n-1}{n}\right\}
$$

and

$$
W(x, y)= \begin{cases}1, & d(x, y) \leq r \\ 0, & \text { otherwise }\end{cases}
$$

where $r \in(0,1 / 2)$ is a fixed parameter, and

$$
d(x, y)=\min \{|x-y|, 1-|x-y|\} .
$$

With these choices of $W$ and $X_{n}, G_{n}=\mathbb{G}\left(W, X_{n}\right)$ is the $\lfloor r n\rfloor$-NN graph, which is used as a starting point in the construction of SW graphs [25, 20].

We are now in a position to interpret SW networks as W-random graphs (cf. [18]). To this end, let

$$
G_{n, p}=\mathbb{G}\left(W_{p}, X_{n}\right), \text { where } W_{p}=(1-p) W+p(1-W), p \in[0,0.5] .
$$

Let us call $(i, j) \in[n]^{2}, i \neq j$, a local edge if $(i, j) \in E\left(C_{n,\lfloor r n\rfloor}\right)$, and a long-range one otherwise. The construction of $G_{n, p}$ can be interpreted as follows: $G_{n, p}$ is obtained from $C_{n,\lfloor r n\rfloor}$ by letting the latter to shed each local connection with probability $p$ and to add each long-range one with the same probability (see Fig. 2p). In particular, $G_{n, 0}$ coincides with $C_{n, \mid r n\rfloor}$, and $G_{n, 0.5}$ is the Erdős-Rényi graph with the edge density $1 / 2$. The construction of $G_{n, p}$ resembles that of the Watts-Strogats SW graphs [25], but differs from it in some details. For other $W$-random graphs, which match the classical Watts-Strogatz and Manasson-Newman-Watts models of SW graphs [25, 20, 19] more closely, we refer an interested reader to [18, Section 5]. In the remainder of this paper, 2.4) will be used as a model of SW connectivity.

Now we turn to the main point of this section: the derivation of the continuum limit for the Kuramoto model on the sequence of SW graphs $\left\{G_{n, p}\right\}$. To explain the intuition behind the limiting procedure, let us rewrite $(1.2)$ as

$$
\frac{d}{d t} u_{n i}=\frac{\sigma}{n} \sum_{j=1}^{n} a_{i j}^{(n)} \sin \left(2 \pi\left(u_{j}-u_{i}\right)\right), i \in[n],
$$

where $\left(a_{i j}^{(n)}\right)$ stands for the $n \times n$ adjacency matrix of $G_{n, p}$. Our goal is to derive the infinite-dimensional analog of 2.5) as $n \rightarrow \infty$. To this end, we define a step-function $u_{n}: I \times \mathbb{R}$ such that

$$
u_{n}(x, t)=u_{n i}(t), x \in\left[(i-1) n^{-1}, i n^{-1}\right), i \in[n],
$$

where $u_{n}=\left(u_{n 1}, u_{n 2}, \ldots, u_{n n}\right)$ is a solution of the discrete model 2.5$)$. We expect that as $n \rightarrow \infty$, the sequence of step-functions $u_{n}(x, t)$ approaches $u(x, t)$ (in the appropriate norm). The latter function is interpreted as the solution for the continuum model.

Next, we have to decide how to interpret the adjacency matrix $\left(a_{i j}^{(n)}\right)$ as $n \rightarrow \infty$. The answer to this question is suggested by the theory of graph limits, which for every sequence of convergent dense graphs, 
like $\left\{G_{n, p}\right\}$, defines the limiting object - a symmetric measurable function on the unit square. The limit of a convergent graph sequence is called a graphon [15]. From the theory of graph limits, we know that $\left\{G_{n, p}\right\}$, as a sequence of $W$-random graphs, converges to the underlying graphon $W_{p}$ (cf. [16]). Below, we provide an informal intuitive interpretation of the graph limit $W_{p}$, which is meant to explain its appearance in the continuum limit of (2.5). A detailed exposition of the theory of graph limits can be found in the monograph by Lovász [15], or in the following papers [5, 16, 23].

To see the meaning of the graph limit $W_{p}$, it is instructive to recall the geometric representation of the adjacency matrices of SW graphs in Fig. 2 b,c. The matrices depicted in these plots correspond to $G_{80,0.2}$ and $G_{400,0.2}$. These graphs belong to the same sequence of SW graphs $\left\{G_{n, 0.2}\right\}$ generated by graphon $W_{0.2}$ with $r=3 / 40$ (see 2.4p). Note that for large $n$, as in Fig. 2k, black pixels fill different regions of the unit square uniformly with two different densities $1-p$ and $p$, just as in the definition of the function $W_{p}$ (see (2.2) and (2.4p). In fact, the graphon $W_{p}$ represents the density of connections between different subsets of nodes of the SW graph $G_{n, p}$ for large $n$.

We have identified $u(x, t)$ and $W_{p}(x, y)$ as continuous counterparts of $u_{n}(t)=\left(u_{n 1}(t), u_{n 2}(t), \ldots, u_{n n}(t)\right)$ and $\left(a_{i j}^{(n)}\right)$ in the large $n$ limit. Furthermore, the right-hand side in 2.5 has the form of a Riemann sum. Therefore, by sending $n \rightarrow \infty$ in 2.5 , we formally arrive at the following evolution equation

$$
\frac{\partial}{\partial t} u(x, t)=\sigma \int_{I} W_{p}(x, y) \sin (2 \pi(u(y, t)-u(x, t))) d y .
$$

The continuum limit (2.7) was rigorously justified in [18]. Specifically, Theorem 3.4 of [18] implies that for any $T>0, u_{n}(x, t)$ (see 2.6) converge to $u(x, t)$ in the $C\left(0, T ; L^{2}(I)\right)$ norm in probability as $n \rightarrow \infty$, provided the initial conditions $u_{n}(\cdot, 0)$ and $u(\cdot, 0)$ are sufficiently close.

\section{The stability analysis of q-twisted states}

The goal of this section is to elucidate the role of $q$-twisted states $(1.3)$ in the long-time asymptotic behavior of solutions of the Kuramoto model (1.2) on SW graphs 2.4) for large $n$. First, we show that $q$-twisted states become steady state solutions of (1.2), 2.4) as $n \rightarrow \infty$ with probability 1 (see Lemma 3.1). Next, we use the continuum limit 2.77 to study the linear stability of the continuous $q$-twisted states

$$
u^{(q)}(x)=q x+c \quad(\bmod 1), c \in[0,1), q \in \mathbb{Z}
$$

(see Theorem 3.3). The results of this section will be used in Section 4 to study synchronization in SW networks.

Note that while the continuous $q$-twisted states (3.1) solve (2.7) for all values of $p$, their discrete counterparts generically do not solve (1.2) for $p>0$. They nonetheless remain relevant for the following reasons. First, for large $n, 1.2$ has solutions that remain close to $u_{n}^{(q)}$ on finite time intervals (cf. [18, Theorem 3.4]). Furthermore, the following lemma shows directly (i.e., without invoking the limiting equation (2.7)) that

$u_{n}^{(q)}$ become the steady-state solutions of 1.2 for $p>0$ in the limit as $n \rightarrow \infty$.

Lemma 3.1. Let $\left\{G_{n, p}\right\}$ be a sequence of $S W$ graphs (2.4), (2.2) with $r \in(0,1 / 2]$ and

$$
\mathcal{R}_{n i}\left(u_{n}\right)=\frac{1}{n} \sum_{j:(i, j) \in E\left(G_{n, p}\right)} \sin \left(2 \pi\left(u_{n j}-u_{n i}\right)\right), i \in[n],
$$


where $u_{n}=\left(u_{n 1}, u_{n 2}, \ldots, u_{n n}\right)$. Then for any $q \in \mathbb{N} \bigcup\{0\}$ and $i \in \mathbb{N}$

$$
\lim _{n \rightarrow \infty} \mathcal{R}_{n i}\left(u_{n}^{(q)}\right)=0
$$

almost surely.

Proof. Assume first that $r \in(0,1 / 2)$. Let $i \in \mathbb{N}$ be arbitrary but fixed and let $n \geq i$. To simplify notation, throughout this proof we suppress the dependence of various sets and variables on $i$. Denote

$$
J_{n}^{(1)}=\{j \in[n]: j \neq i \& d(i, j) \leq r n\}, J_{n}^{(2)}=\{j \in[n]: j \neq i \& d(i, j)>r n\},
$$

$n_{1}:=\left|J_{n}^{(1)}\right|=2\lfloor r n\rfloor$ and $n_{2}=n-1-n_{1}$. Note that for $r \in(0,0.5), n_{1,2}$ tend to infinity with $n \rightarrow \infty$.

Let $\left\{\xi_{n j}^{(k)}\right\}_{j \in J_{n}^{(1)}}, k \in\{1,2\}$ be two arrays of independent identically distributed (IID) random variables (RVs) such that

$$
\mathcal{L}\left(\xi_{n 1}^{(1)}\right)=\operatorname{Bin}(1-p) \text { and } \mathcal{L}\left(\xi_{n 1}^{(2)}\right)=\operatorname{Bin}(p) .
$$

Here, $\mathcal{L}(\cdot)$ stands for the probability distribution of a $\mathrm{RV}$, and $\operatorname{Bin}(p)$ denotes the binomial distribution with parameter $p$. Define

$$
\eta_{n j}^{(k)}=\xi_{n j}^{(k)} \sin \left(2 \pi\left(u_{n j}^{(q)}-u_{n i}^{(q)}\right)\right)=\xi_{n j}^{(k)} \sin \left(\frac{2 \pi q(j-i)}{n}\right), j \in J_{n}^{(k)}, k \in\{1,2\} .
$$

Using 3.2 and the definitions of $\left\{\eta_{n j}^{(k)}\right\}, k \in\{1,2\}$, we have

$$
\mathcal{R}_{n i}\left(u^{(q)}\right)=\frac{n_{1}}{n}\left(n_{1}^{-1} S_{n}^{(1)}\right)+\frac{n_{2}}{n}\left(n_{2}^{-1} S_{n}^{(2)}\right),
$$

where

$$
S_{n}^{(k)}=\sum_{j \in J_{n}^{(k)}} \eta_{n j}^{(k)}, k \in\{1,2\} .
$$

Using (3.4) and (3.5), we estimate

$$
\begin{aligned}
& \mathbb{E} \eta_{n j}^{(k)^{2}}=\sin \left(\frac{2 \pi q(j-i)}{n}\right)^{2} p_{k} \leq 1, \\
& \mathbb{E} \eta_{n j}^{(k)^{4}}=\sin \left(\frac{2 \pi q(j-i)}{n}\right)^{4} p_{k} \leq 1, k \in\{1,2\}, j \in[n],
\end{aligned}
$$

where $p_{1}=1-p$ and $p_{2}=p$.

Further, using (3.4) and 3.7), we have

$$
\mathbb{E} S_{n}^{(k)}=p_{k} \sum_{j \in J_{n}^{(k)}} \sin \left(\frac{2 \pi q(j-i)}{n}\right)=0, k \in\{1,2\},
$$

because $J_{n}^{(k)}$ is symmetric about $i$. 
From this point, the proof follows the lines of the proof of the strong law of large numbers [4, Theorem 6.1]. Specifically, we estimate

$$
\begin{aligned}
\mathbb{E} S_{n}^{(k)^{4}} & =\sum_{j_{1}, j_{2}, j_{3}, j_{4} \in J_{n}^{(k)}} \mathbb{E}\left[\eta_{n j_{1}}^{(k)} \eta_{n j_{2}}^{(k)} \eta_{n j_{2}}^{(k)} \eta_{n j_{2}}^{(k)}\right] \\
& =\sum_{j \in J_{n}^{(k)}} \mathbb{E}\left[\eta_{n j}^{(k)^{4}}\right]+\left(\begin{array}{l}
4 \\
2
\end{array}\right) \sum_{\substack{j_{1}<j_{2}, j_{1}, j_{2} \in J_{n}^{(k)}\\
}} \mathbb{E}\left[\eta_{n j_{1}}^{(k)^{2}} \eta_{n j_{2}}^{(k)^{2}}\right] \\
& \leq n+3 n(n-1)<3 n^{2}, k \in\{1,2\},
\end{aligned}
$$

where we used independence of $\left\{\eta_{n j}^{(k)}: j \in J_{n}^{(k)}\right\}, 3.8,3.9$, and 3.10 .

By Markov inequality, using (3.10) and 3.11, for any $\epsilon>0$ we have

$$
\mathbb{P}\left\{\left|S_{n}^{(k)}\right| \geq n \epsilon\right\} \leq 3 \epsilon^{-4} n^{-2},
$$

which in turn implies via the first Borel-Cantelli lemma [4, Theorem 4.3]

$$
\mathbb{P}\left\{\left|n_{k}^{-1} S_{n}^{(k)}\right| \geq \epsilon \text { holds for infinitely many } n\right\}=0, k \in\{1,2\} .
$$

By Theorem 5.2(i) in [4], the last statement is equivalent to convergence of $n_{k}^{-1} S_{n}^{(k)}$ to 0 almost surely. Therefore, the combination of 3.6,

$$
\lim _{n \rightarrow \infty} \frac{n_{1}}{n}=2 r, \text { and } \lim _{n \rightarrow \infty} \frac{n_{2}}{n}=1-2 r,
$$

yields 3.3 for $r \in(0,1 / 2)$. If $r=1 / 2$ then $S_{n}^{(2)}=0$, and 3.3 follows by repeating the above argument for $S_{n}^{(1)}$ only.

Having shown, that $q$-twisted states solve the Kuramoto equation on $\left\{G_{n, p}\right\}$ in the large $n$ limit, we turn to study the stability of $q$-twisted states, as steady state solutions of the continuum equation. For concreteness, in the remainder of this paper we restrict to the attractive coupling case, i.e., $\sigma=1$ in (1.2). The repulsive coupling case can be treated similarly. Thus, we rewrite 2.7) as follows

$$
\frac{\partial u(x, t)}{\partial t}=\int_{I} K_{p}(y-x) \sin (2 \pi(u(y, t)-u(x, t))) d y
$$

where

$$
K_{p}(x)=p G_{1 / 2}(x)+(1-2 p) G_{r}(x), \quad G_{r}(x)= \begin{cases}1, & d(x)<r \\ 0, & \text { otherwise }\end{cases}
$$

and $d(\cdot)$ was defined in 2.3 .

Remark 3.2. The results of this section hold for a more general class of kernels $K_{p}$. It is sufficient to assume that $K_{p}(\cdot)$ is a even bounded function.

We are now in a position to formulate a sufficient condition for stability of $q$-twisted states $u^{(q)}$ (see (3.1). 
Theorem 3.3. For $q \in \mathbb{Z}$ and $p \in[0,0.5]$, the $q$-twisted state $u^{(q)}$ is a steady state of 3.13 . Moreover, it is linearly stable with respect to perturbations from $L^{\infty}(I)$ provided

$$
\lambda_{p}(q, m):=\tilde{K}_{p}(m+q)-2 \tilde{K}_{p}(q)+\tilde{K}(q-m)<0 \forall m \in \mathbb{N},
$$

where

$$
\tilde{K}_{p}(m)=\int_{I} K_{p}(x) \cos (2 \pi m x), m \in \mathbb{Z}
$$

Remark 3.4. Theorem 3.3 extends the sufficient condition that was obtained for $k$-NN deterministic networks in [26] to networks on SW graphs.

We precede the proof of Theorem 3.3 with the following observation, which follows from the oddsymmetry of the product $K_{p}(x) \sin (x)$.

Lemma 3.5. Let $\mathbf{u} \in C^{1}\left(\mathbb{R}, L^{\infty}(I)\right)$ be a solution of 3.13). Then

$$
\frac{\partial}{\partial t} \int_{I} u(x, t) d x=0
$$

Proof. By integrating both parts of (3.13) over $I$ and using Fubuni's theorem, we have

$$
\frac{\partial}{\partial t} \int_{I} u(x, t) d x=\int_{I \times I} K_{p}(y-x) \sin (2 \pi(u(y, t)-u(x, t))) d x d y,
$$

where we also interchanged differentiation with integration on the left-hand side, because $\mathbf{u} \in C^{1}\left(\mathbb{R}, L^{\infty}(I)\right)$ and $\mathbf{u}^{\prime}$ is bounded on $\mathbb{R}$ (cf. [17, Theorem 3.1]). The integral on the right-hand side is zero, because the domain of integration $I \times I$ is symmetric with respect to the line $y=x$ and the integrand is an odd symmetric function.

Proof. (Theorem 3.3) Consider $H=L^{\infty}(I)$ as a subspace of $L^{2}(I)$. Then

$$
H=H_{0} \oplus H_{0}^{\perp},
$$

where

$$
H_{0}=\left\{u \in H: \int_{I} u(x) d x=0\right\} \text { and } H_{0}^{\perp}=\{u \in H: u(x)=c \text { a.e., for some } c \in \mathbb{R}\} .
$$

By Lemma 3.5, $H_{0}$ and $H_{0}^{\perp}$ are invariant under the flow 2.7). It is easy to see that $q$-twisted state solutions of 2.7 remain stable under spatially homogeneous perturbations from $H_{0}^{\perp}$. Therefore, it is sufficient to study the stability of $q$-twisted states with respect to the perturbations from $H_{0}$.

To this end, we linearize 3.13 about the $q$-twisted state $u^{(q)}$, i.e., we plug $u(x, t)=u^{(q)}(x)+\eta(x, t)$ into 3.13 and retain only the linear terms on the right-hand side of the resultant equation

$$
\frac{\partial \eta(x, t)}{\partial t}=2 \pi \int_{I} K_{p}(y-x) \cos (2 \pi q(y-x))[\eta(y, t)-\eta(x, t)] d y
$$


which we supply with the initial condition

$$
\eta(\cdot, 0) \in H_{0}
$$

We expand $\eta(x, t)$ in the Fourier series

$$
\eta(x, t)=\sum_{k \in \mathbb{Z}} \hat{\eta}_{k}(t) e^{-i 2 \pi k x}, \text { where } \hat{\eta}_{k}(t)=\int_{I} \eta(x, t) e^{i 2 \pi k x} d x .
$$

Note that $\hat{\eta}_{0} \equiv 0$, because $\hat{\eta}_{0}(0)=0$ as follows from 3.20).

To simplify notation we rescale the time variable in (3.19) to absorb the $2 \pi$ factor on the right-hand side. Thus, 3.19) can be rewritten in the following form

$$
\frac{\partial \eta}{\partial t}=K_{p}^{(q)} * \eta-\left(\operatorname{Re} \hat{K}_{p}(q)\right) \eta
$$

where $*$ denotes the convolution and

$$
K_{p}^{(q)}(x)=K_{p}(x) \cos (2 \pi q x), \hat{K}_{p}(m)=\int_{I} K_{p}(x) e^{i 2 \pi m x} d x
$$

By applying the Fourier transform to both sides of 3.22, we have

$$
\frac{d \hat{\eta}_{m}}{d t}=\left[\hat{K}_{p}^{(q)}(m)-\operatorname{Re} \hat{K}_{p}(q)\right] \hat{\eta}_{m}, m \in \mathbb{Z} /\{0\}
$$

Thus, using Parseval's identity, we arrive at the sufficient condition for linear stability of the $q$-twisted state

$$
\operatorname{Re}\left[\hat{K}_{p}^{(q)}(m)-\hat{K}_{p}(q)\right]<0 \forall m \in \mathbb{Z} /\{0\}
$$

Further, it is easy to verify that

$$
\hat{K}_{p}^{(q)}=\frac{\hat{K}_{p}(m+q)+\hat{K}_{p}(m-q)}{2}, m \in \mathbb{Z} /\{0\} .
$$

Thus, 3.24 can be rewritten as follows

$$
\lambda_{p}(q, m)=\tilde{K}_{p}(m+q)-2 \tilde{K}_{p}(q)+\tilde{K}_{p}(m-q)<0 \forall m \in \mathbb{Z} /\{0\},
$$

where

$$
\tilde{K}_{p}(m)=\operatorname{Re} \hat{K}_{p}(m)=\int_{I} K_{p}(x) \cos (2 \pi m x) d x, m \in \mathbb{Z}
$$

Since $\tilde{K}_{p}(m)$ is an even function, it is sufficient to restrict 3.25 for $m \in \mathbb{N}$. This concludes the proof of Theorem 3.3 . 


\section{Synchronization}

In this section, we apply Theorem 3.3 to study synchronization in the Kuramoto model on SW graphs (3.13). Throughout this section, we continue to use the rescaled linearized equation (3.22).

Using (3.14) and (3.16), we have

$$
\tilde{K}_{p}(m)=p \tilde{G}_{1 / 2}(m)+(1-2 p) \tilde{G}_{r}(m),
$$

where

$$
\tilde{G}_{r}(m)=\left\{\begin{array}{cc}
2 r S(2 \pi m r), & m \neq 0, \\
2 r, & m=0,
\end{array}\right.
$$

and $S(x)=x^{-1} \sin x$.

By applying Theorem 3.3 to 3.13 with 3.14 , we obtain the following condition for stability of the $q$-twisted states

$$
\lambda_{p}(q, m)=p D_{m}^{2} \tilde{G}_{1 / 2}(q)+(1-2 p) D_{m}^{2} \tilde{G}_{r}(q)<0 \forall m \in \mathbb{N},
$$

where $D_{m}^{2}$ stands for the second-order difference

$$
D_{m}^{2} \tilde{K}(q)=\tilde{K}_{p}(q+m)-2 \tilde{K}_{p}(q)+\tilde{K}_{p}(q-m) .
$$

Using (4.1), we rewrite (4.2) as follows

$$
\lambda_{p}(q, m)=p\left(-2 \delta_{q 0}+\delta_{q m}\right)+(1-2 p) \lambda_{0}(q, m)<0 \forall m \in \mathbb{N},
$$

where

$$
\lambda_{0}(q, m)=2 r[S(2 \pi r(q+m))-2 S(2 \pi r q)+S(2 \pi r(q-m))]
$$

and the Kronecker delta $\delta_{i j}=1$ if $i=j$ and is equal to 0 otherwise.

Equation (4.4) yields the following sufficient condition for synchronization in SW networks

$$
\lambda_{p}(0, m)=-2 p+(1-2 p) \lambda_{0}(0, m)<0 \forall m \in \mathbb{N},
$$

where

$$
\lambda_{0}(0, m)=4 r(S(2 \pi m r)-1) .
$$

Conditions (4.4) and (4.5) show how the random long-range connections in the SW network affect the stability of the $q$-twisted states. For the $k$-NN coupled networks, the linear stability of the $q$-twisted states was studied in detail by Wiley, Strogatz, and Girvan [26]. They found that the $q$-twisted state is stable provided $0 \leq q r<\mu$, where $\mu \approx 0.66$ is a solution of a certain transcendental equation (see [26, Equation (18)]). For these values of $q$, we have

$$
\lambda_{0}(q, m)<0 \forall m \in \mathbb{N} .
$$

Thus, the synchronous solution $(q=0)$ is stable for the full range of $r \in(0,0.5)$. In addition, for small $r>0$, there may be many other stable $q$-twisted states. For such values of $r$, these $q$-twisted states remain stable on SW graphs $G_{n, p}$ with

$$
0<p<\frac{-\lambda_{0}(q, q)}{1-2 \lambda_{0}(q, q)}
$$


From Equation 4.5 we find that the synchronous state remains stable for all $p \in(0,0.5]$. Moreover, for $p>0$, Equation (4.5) provides a relaxed condition compared to the corresponding conditions for $\lambda_{0}(0, m)$. In contrast, from Equation (4.4) we find that for increasing values of $p$ the conditions for stability become more stringent. In particular, 4.7) shows that a $q$-twisted state, which is stable for $p=0$, may lose stability at some positive value of $p$. From this, we conclude that long-range random connections promote synchrony, while inhibiting other $q$-twisted states.

Next, we discuss the stability of the synchronous regime in SW networks in a little more detail. To this end, we employ the leading exponent

$$
\lambda^{*}(p)=\max _{m \in \mathbb{N}} \lambda_{p}(0, m)=\lambda_{p}(0,1)
$$

which reflects the rate of convergence to the synchronous state. Larger values of $\left|\lambda^{*}(p)\right|$ indicate stronger synchronization. For small values of the coupling range $r>0$, from 4.5), we estimate

$$
\lambda^{*}(0)=\frac{-16 \pi^{2}}{3} r^{3}+O\left(r^{5}\right) .
$$

Equation (4.9) shows that larger values of $r$ result in faster synchronization. Furthermore, from (4.5) and (4.9), we estimate the leading exponent for SW connectivity

$$
\lambda^{*}(p)=-2 p+(1-2 p) \lambda^{*}(0) .
$$

Since for small $r>0, \lambda^{*}(0)>-1$, we have

$$
\frac{d}{d p} \lambda^{*}(p)=-2\left(1+\lambda^{*}(0)\right)<0
$$

i.e., for increasing values of $p$ the leading exponent $\left|\lambda^{*}(p)\right|$ grows. This once again shows that adding the long-range random connections helps synchronization in the Kuramoto model on SW graphs.

\section{Continuation}

In this section, we develop a numerical continuation method, which will be used to study the impact of the long-range random connections on the spatial structure of the stationary solutions of the Kuramoto model (1.2).

\subsection{Preliminaries}

First, we adjust our notation to the purpose of this section. To this end, we rewrite $(1.2)$ as follows

$$
\dot{u}_{i}=n^{-1} \sum_{j=1}^{n} a_{i j}(p) \sin \left(2 \pi\left(u_{j}-u_{i}\right)\right), i \in[n],
$$


where $u=\left(u_{1}, u_{2}, \ldots, u_{n}\right)$ and $n \in \mathbb{N}$. Matrix $A(p)=\left(a_{i j}(p)\right)$ is an $n \times n$ adjacency matrix of the $\mathrm{SW}$ graph $G_{n, p}$ with the randomization parameter $p \in[0,0.5]$. Below we will use the $\ell_{1}$-norm of matrix $A=\left(a_{i j}\right) \in \mathbb{R}^{n \times n}$

$$
\|A\|_{1}=n^{-2} \sum_{i, j=1}^{n}\left|a_{i j}\right| .
$$

The vector form of (5.1) is given by

$$
\dot{u}=F(u, p),
$$

where $F: \mathbb{R}^{n} \times \mathbb{R} \rightarrow \mathbb{R}^{n}$. Recall that for any $q \in \mathbb{Z}, q$-twisted state

$$
u_{q}=q x \quad(\bmod 1), x:=\left(0, n^{-1}, 2 n^{-1}, \ldots,(n-1) n^{-1}\right)
$$

is a stationary solution of 5.2 with $p=0$, i.e.,

$$
F\left(u_{q}, 0\right)=0 .
$$

Thus, one can try to continue from $u_{q}$ for $p$ near 0 to see how the addition of new random edges affects the structure of the steady state solutions of (5.2). However, one immediately runs into the following problem: in general, $F(\cdot, p)$ is not (stochastically) continuous in $p$, as the following lemma shows.

Lemma 5.1. Let $G_{n, p+\Delta p}$ and $G_{n, p}$ be two independent copies of $S W$ graphs with randomization parameters $0<p+\Delta p, p<0.5$. Then

$$
\mathbb{E}\|A(p+\Delta p)-A(p)\|_{1}=2\left(1-\frac{1}{n}\right) \alpha(p),
$$

where

$$
\alpha(p)=2 p(1-p)+\Delta p(1-2 p)=O(1),
$$

and $A(p+\Delta p)$ and $A(p)$ stand for the adjacency matrices of $G_{n, p+\Delta p}$ and $G_{n, p}$ respectively.

Proof. Let

$$
L=\left\{(i, j) \in[n]^{2}: 0<\min \{|i-j|, n-|i-j|\} \leq k\right\}
$$

be the set of indices corresponding to the edges of $k$-NN graph, and let $L^{c}=[n]^{2} / L$ be its complement.

Denote RVs

$$
\xi_{i j}=\left|a_{i j}(p+\Delta p)-a_{i j}(p)\right|, 1 \leq i<j \leq n .
$$

We show that $\left\{\xi_{i j}\right\}$ are IID binomial RVs. Indeed, let $1 \leq i<j \leq n$ and $(i, j) \in L$. Then

$$
\begin{aligned}
\mathbb{P}\left\{\xi_{i j}=1\right\} & =\mathbb{P}\left\{\left(a_{i j}(p+\Delta p)=1\right) \&\left(a_{i j}(p)=0\right)\right\}+\mathbb{P}\left\{\left(a_{i j}(p+\Delta p)=0\right) \&\left(a_{i j}(p)=1\right)\right\} \\
& =(1-p-\Delta p) p+(p+\Delta p)(1-p)=\alpha(p) .
\end{aligned}
$$

Likewise, for $1 \leq i<j \leq n$ and $(i, j) \in L^{c}$, we have

$$
\begin{aligned}
\mathbb{P}\left\{\xi_{i j}=1\right\} & =\mathbb{P}\left\{\left(a_{i j}(p+\Delta p)=1\right) \&\left(a_{i j}(p)=0\right)\right\}+\mathbb{P}\left\{\left(a_{i j}(p+\Delta p)=0\right) \&\left(a_{i j}(p)=1\right)\right\} \\
& =(p+\Delta p)(1-p)+(1-p-\Delta p) p=\alpha(p) .
\end{aligned}
$$



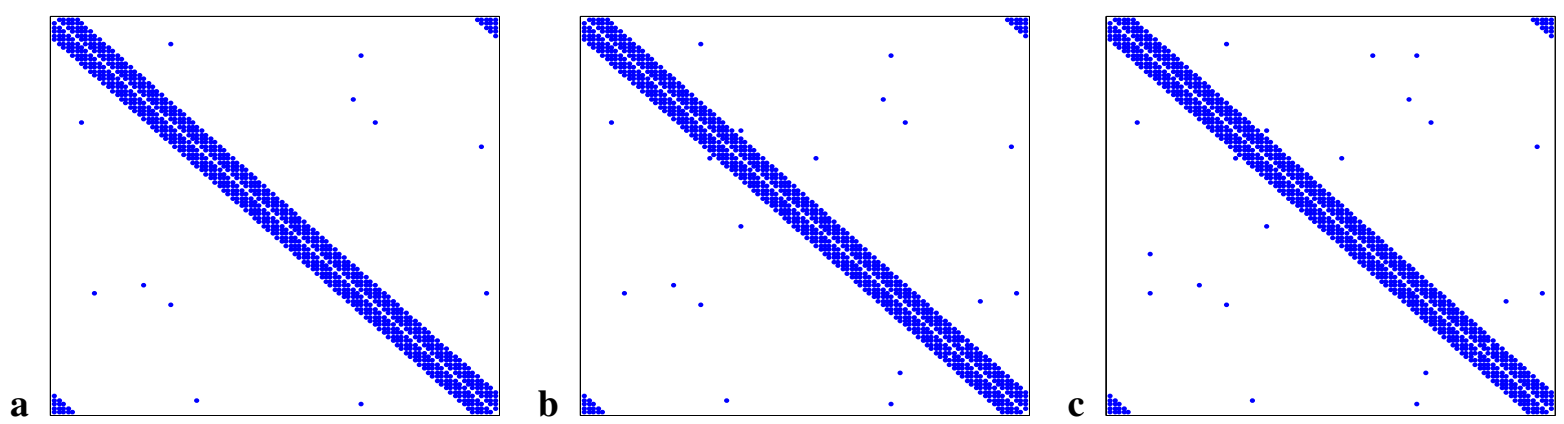

Figure 3: Schematic representation of the adjacency matrices of three consistent SW graphs $G_{n, p}$ generated using (5.9)-(5.12): $n=100, p=0.001$ (a), $p=0.002(\mathbf{b}), p=0.003$ (c). Black dots denote entries of the adjacency matrix that are equal to 1 .

Thus, $\mathcal{L}\left(\xi_{i j}\right)=\operatorname{Bin}(\alpha(p)), 1 \leq i<j \leq n$, and

$$
\mathbb{E}\|A(p+\Delta p)-A(p)\|_{1}=\frac{2}{n^{2}} \mathbb{E}\left(\sum_{1 \leq i<j \leq n} \xi_{i j}\right)=\frac{2 n(n-1)}{n^{2}} \alpha(p) .
$$

Remark 5.2. Note that for $p \in(0,1 / 2), \mathbb{E}\|A(p+\Delta p)-A(p)\|_{1}$ stays bounded away from 0 as $\Delta p \rightarrow 0$.

Remark 5.3. Using the strong law of large numbers, one can show that

$$
\lim _{n \rightarrow \infty}\|A(p+\Delta p)-A(p)\|_{1}=2 \alpha(p) \quad \text { almost surely. }
$$

Indeed, since $\left\{\xi_{i j}\right\}_{1 \leq i<j \leq n}$ are IID binomial RVs, $\mathcal{L}\left(\xi_{12}\right)=\operatorname{Bin}(\alpha(p))$, by the strong law of large numbers, we have

$$
\mathbb{P}\left\{\lim _{n \rightarrow \infty} \frac{1}{n(n-1)} \sum_{1 \leq i<j \leq n} \xi_{i j}=\alpha(p)\right\}=1 .
$$

Then (5.5) follows from 5.6, because

$$
\|A(p+\Delta p)-A(p)\|_{1}=2\left(1-\frac{1}{n}\right) \frac{1}{n(n-1)} \sum_{1 \leq i<j \leq n} \xi_{i j} .
$$

\subsection{Consistent family of SW graphs}

Lemma 5.1 shows that $F(u, p)$ is not stochastically continuous in $p$. Therefore, nonlinear equation

$$
F(u, p)=0,
$$

as it stands can not be used for continuation. To rectify this problem, we introduce a special family of SW graphs, along which $F(u, p)$ is stochastically continuous in $p$. 
Definition 5.4. Let $N \gg 1$ and $h=2 N^{-1}$. Consider a family of $S W$ graphs $\left\{G_{n, p_{k}}\right\}_{k=0}^{N}$, where $p_{k}=k h$. Graphs $G_{n, p_{k}}$ are said to form a consistent family of $S W$ graphs if

$$
\mathbb{E}\left\|A\left(p_{k+1}\right)-A\left(p_{k}\right)\right\|_{1}=O(h) \quad \forall k \in\{0,1,2, \ldots, N-1\} .
$$

It is not hard to construct a consistent family of SW graphs. This can be done inductively. Suppose $G_{n, p_{k}}$ is given for some $0 \leq k \leq N-1$. Then $G_{n, p_{k+1}}$ is a random graph defined by the following conditions. For $1 \leq i<j \leq n$ and $(i, j) \in L$, we set

$$
\begin{aligned}
& \mathbb{P}\left\{a_{i j}\left(p_{k+1}\right)=0 \mid a_{i j}\left(p_{k}\right)=1\right\}=q_{k}, q_{k}:=h\left(1-p_{k}\right)^{-1}, \\
& \mathbb{P}\left\{a_{i j}\left(p_{k+1}\right)=0 \mid a_{i j}\left(p_{k}\right)=0\right\}=1 .
\end{aligned}
$$

Likewise, for $1 \leq i<j \leq n$ and $(i, j) \in L^{c}$, we have

$$
\begin{aligned}
& \mathbb{P}\left\{a_{i j}\left(p_{k+1}\right)=1 \mid a_{i j}\left(p_{k}\right)=1\right\}=1, \\
& \mathbb{P}\left\{a_{i j}\left(p_{k+1}\right)=1 \mid a_{i j}\left(p_{k}\right)=0\right\}=q_{k} .
\end{aligned}
$$

It is easy to check that $A\left(p_{k+1}\right)$ defines a SW graph $G_{n, p_{k+1}}$. Moreover, graphs $G_{n, p_{k}}$ constructed above form a consistent family.

Lemma 5.5. Recursive relations (5.9)-(5.12) define a consistent family of SW graphs.

Proof. Let $k \in\{0,1, \ldots, N-1\}$ be arbitrary and define

$$
\xi_{i j}=\left|a_{i j}\left(p_{k+1}\right)-a_{i j}\left(p_{k}\right)\right|, 1 \leq i<j \leq n .
$$

We show that $\mathcal{L}\left(\xi_{i j}\right)=\operatorname{Bin}(h)$. Indeed, let $(i, j) \in L$. Then

$$
\begin{aligned}
\mathbb{P}\left\{\xi_{i j}=1\right\} & =\mathbb{P}\left\{a_{i j}\left(p_{k+1}\right) \neq a_{i j}\left(p_{k}\right)\right\}=\mathbb{P}\left\{\left(a_{i j}\left(p_{k+1}\right)=0\right) \&\left(a_{i j}\left(p_{k}\right)=1\right)\right\} \\
& =\mathbb{P}\left\{a_{i j}\left(p_{k+1}\right)=0 \mid a_{i j}\left(p_{k}\right)=1\right\} \mathbb{P}\left\{a_{i j}\left(p_{k}\right)=1\right\}=q_{k}\left(1-p_{k}\right)=h .
\end{aligned}
$$

Likewise, for $1 \leq i<j \leq n$ and $(i, j) \in L^{c}$, we have

$$
\begin{aligned}
\mathbb{P}\left\{\xi_{i j}=1\right\} & =\mathbb{P}\left\{a_{i j}\left(p_{k+1}\right) \neq a_{i j}\left(p_{k}\right)\right\}=\mathbb{P}\left\{\left(a_{i j}\left(p_{k+1}\right)=1\right) \&\left(a_{i j}\left(p_{k}\right)=0\right)\right\} \\
& =\mathbb{P}\left\{a_{i j}\left(p_{k+1}\right)=1 \mid a_{i j}\left(p_{k}\right)=0\right\} \mathbb{P}\left\{a_{i j}\left(p_{k+1}\right)=0\right\}=q_{k}\left(1-p_{k}\right)=h
\end{aligned}
$$

Similarly, we compute $\mathbb{P}\left\{\xi_{i j}=1\right\}=1-h, 1 \leq i<j \leq n$. Thus,

$$
\mathbb{E}\left\|A\left(p_{k+1}\right)-A\left(p_{k}\right)\right\|_{1}=\frac{2 n(n-1)}{n^{2}} h=2\left(1-n^{-1}\right) h .
$$

Figure 3 presents several representative examples of SW graphs from a consistent family, which where computed using (5.9)- 5.12). Note that the adjacency matrices in Fig. 3 change little for small changes in the randomization parameter $p$. 

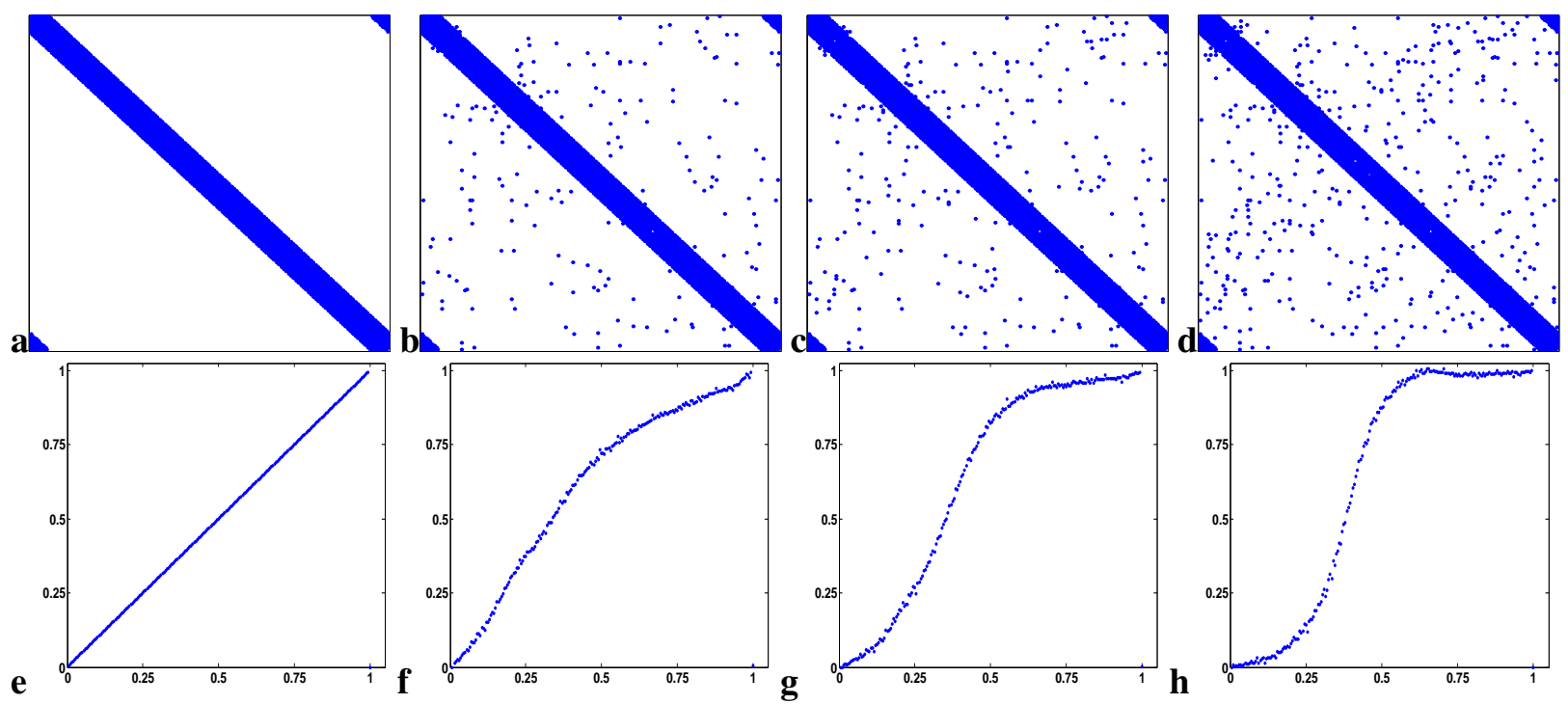

Figure 4: A consistent family of the SW graphs $G_{100, p}(\mathbf{a}-\mathbf{d})$. The corresponding steady-state solutions (5.1) obtained by continuation from 1-twisted state solution (e) are shown in (f-h). The corresponding values of $p$ are $3.5 \cdot 10^{-3}(\mathbf{f}), 4.9 \cdot 10^{-3}(\mathbf{g})$, and $9.9 \cdot 10^{-3}(\mathbf{h})$.
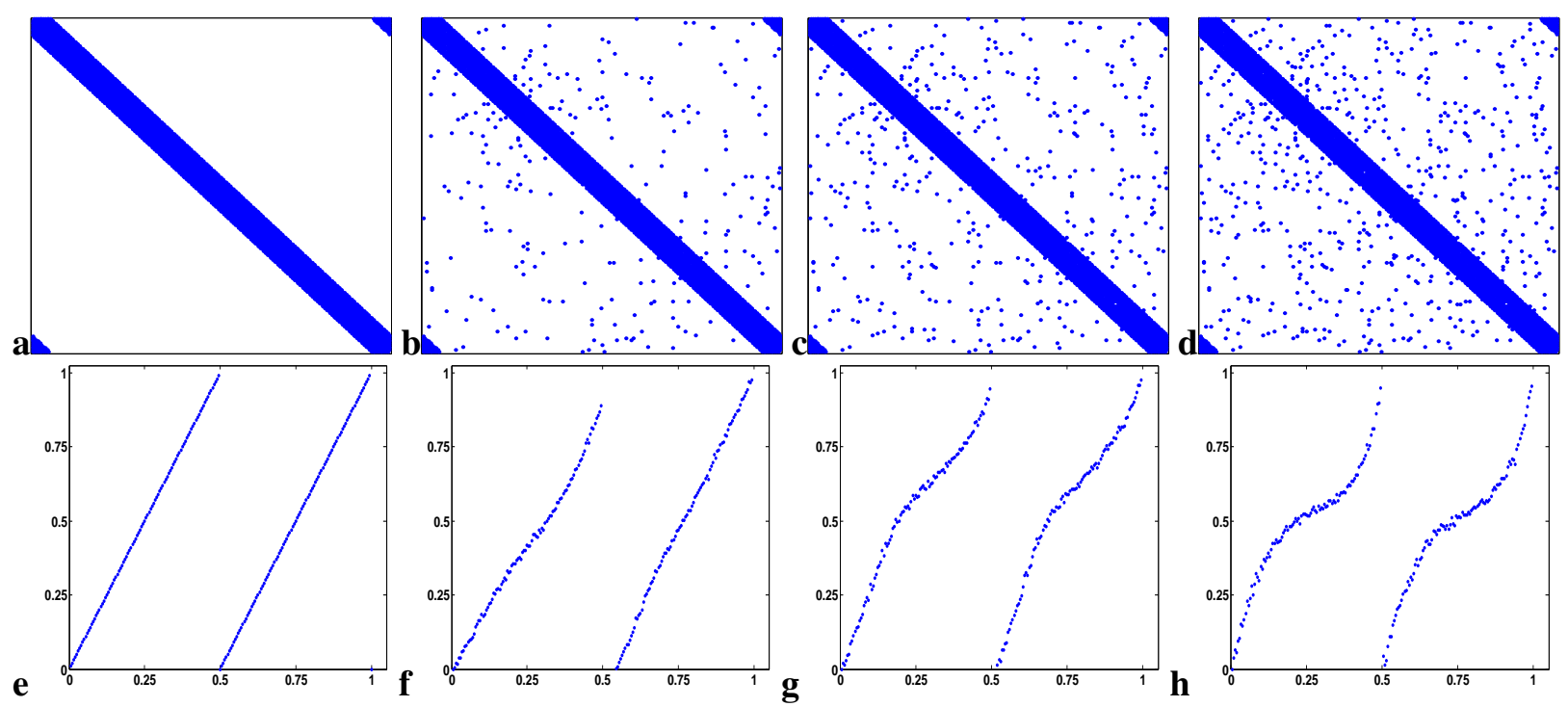

Figure 5: A consistent family of the SW graphs $G_{200, p}$ (a-d). The corresponding steady-state solutions (5.1) obtained by continuation from 2-twisted state solution (e) are shown in (f-h). The corresponding values of $p$ are $6 \cdot 10^{-3}(\mathbf{f}), 1.1 \cdot 10^{-3}(\mathbf{g})$, and $1.63 \cdot 10^{-2}(\mathbf{h})$. 


\subsection{The Newton's continuation and numerical results}

With the idea of the consistent family of SW graphs at hand, we can now set up a numerical continuation procedure based on Newton's method.

Let $h>0$ be sufficiently small and $\left\{G_{n, p_{k}}\right\}_{k=0}^{N}, N=\left\lfloor(2 h)^{-1}\right\rfloor$ be a consistent family of SW graphs (cf. (5.9)-(5.12). Then the right hand side of (5.1) depends continuously on $p$. Indeed, from (5.1) using the definition of the $\ell_{1}$-norm we have

$$
\left\|F\left(u, p_{k+1}\right)-F\left(u, p_{k}\right)\right\|_{1} \leq\left\|A\left(p_{k+1}\right)-A\left(p_{k}\right)\right\|_{1},
$$

where by $\ell_{1}$-norm of vector $F=\left(F_{1}, F_{2}, \ldots, F_{n}\right)$ we mean

$$
\|F\|_{1}=n^{-1} \sum_{i=1}^{n}\left|F_{i}\right| .
$$

From 5.13 and Lemma 5.5, we have

$$
\mathbb{E}\left\|F\left(u, p_{k+1}\right)-F\left(u, p_{k}\right)\right\|_{1} \leq \mathbb{E}\left\|A\left(p_{k+1}\right)-A\left(p_{k}\right)\right\|_{1}=O(h)
$$

uniformly in $u \in \mathbb{R}^{n}$.

To compute $\tilde{u}\left(p_{k+1}\right)$, the approximation of $u\left(p_{k+1}\right)$, the steady state solution of the Kuramoto model 5.1) on the SW graph $G_{n, p_{k+1}}$, we use Newton's iterations

$$
u^{(k+1)}=u^{(k)}-\left[\frac{\partial}{\partial u} F(u(p), p+\Delta p)\right]^{+} F\left(u^{(k)}, p+\Delta p\right),
$$

until the error $\left\|u^{(k+1)}-u^{(k)}\right\|$ gets sufficiently small. To initialize 5.14 , we use $u^{(0)}=\tilde{u}\left(p_{k}\right)$, the approximation of the steady-state solution of (5.1) on $G_{n, p_{k}}$, which was computed at the previous step of the continuation algorithm. In 5.14, the Moore-Penrose pseudo-inverse of the Jacobian matrix $\left[\frac{\partial}{\partial u} F(u(p), p)\right]^{+}$ is used, because

$$
\text { ker } \frac{\partial}{\partial u} F(u(p), p)=\operatorname{span}\left\{(1,1, \ldots, 1)^{\top}\right\} .
$$

The singularity of the Jacobian matrix is due to the translational invariance of solutions of [5.1.

The results of the numerical continuation from $q$-twisted states are shown in Figs 4 and 5 . They highlight the importance of the structure of the network in shaping the spatial patterns. Note that the change of approximately $1 \%$ of the entries in the $\{0,1\}$-valued adjacency matrix was sufficient to transform the 1 twisted state shown in Fig. 4 4 to the step-like solution in Fig. 4h. The numerics also reveal an unexpected effect on the spatial organization of the steady states of the Kuramoto model caused by replacing local connections with long-range random ones. It is best seen from the numerical experiments for 1-twisted states shown in Fig. 4. Note that for increasing values of $p$, the spatial profile develops an interface with progressively increasing slope. We observed the same result for all random realizations of the consistent families of SW networks that we used in these experiments. Furthermore, this effect persists for $q$-twisted states with larger values of $q$. For example, the solution continued from the 2-twisted state shown in Fig. 5 has two plateaus separated by two interfaces. 


\section{Concluding remarks}

In this paper, we studied coupled Kuramoto oscillators on SW graphs. We showed that in the limit as the number of oscillators tends to infinity the Kuramoto equation on SW graphs has a family of $q$-twisted state solutions, like those that have been studied for the networks with $k$-NN coupling [26, 10]. We used the continuum limit for the Kuramoto model on SW graphs to study the stability of the $q$-twisted states. The linear stability analysis showed that the randomization of the network connectivity promotes synchronization, while inhibiting other $q$-twisted states.

To study the role of the long-range connections in shaping the spatial structure of solutions, we developed a continuation method, which reveals the transformation of $q$-twisted state solutions as the randomization parameter is varied. The continuation showed that adding random long-range connections results in spatial patterns composed of plateaus separated by interfaces. The appearance of plateaus is another manifestation of the synchronizing effect of the long range connections. The solutions evolving from the $q$-twisted states with $q \neq 0$ can not become completely synchronous, because their nonzero degree is preserved under continuous deformation. This leads to the formation of interfaces. This scenario is different from known mechanisms of interface formation in scalar reaction-diffusion systems, where the sharpness of the interface is controlled by a small parameter [9], nor does it involve higher order differential operators as in the CahnHilliard equation [7]. Instead, the present mechanism is based on the properties of coupled systems with nonlocal interactions [17]. The kernel (2.4) for the continuum limit (3.13) corresponding to the SW networks is a piecewise continuous function on $I^{2}$. Theorem 3.2 in [17] does not exclude discontinuous in $x$ solutions of $(3.13)$. In particular, the sequences shown in Figs. 4 and 5 may be approaching such discontinuous solutions, and may develop sharp interfaces in the process. As a related observation, we note that spatial patterns shown in Figs. 4 and 5 combine regions of synchronous and asynchronous behaviors when viewed in the original coordinates (see $(1.1)$ with $\omega_{i}=\omega \neq 0, i \in[n]$ ). In this respect, they are similar to the chimera states [12]. Therefore, the results of this work elucidate pattern-formation mechanisms in nonlocally coupled dynamical systems.

Acknowledgements. This work was supported in part by the NSF grant DMS 1109367. 


\section{References}

[1] Daniel M. Abrams and Steven H. Strogatz, Chimera states in a ring of nonlocally coupled oscillators, Internat. J. Bifur. Chaos Appl. Sci. Engrg. 16 (2006), no. 1, 21-37. MR 2214910 (2006k:37043)

[2] M. Barahona and L.M. Pecora, Synchronization in small-world systems, Phys. Rev. Lett. 89 (2002), 054101.

[3] D.S. Bassett and E. Bullmore, Small-world brain networks, Neuroscientist 12(6) (2006), 512-23.

[4] Patrick Billingsley, Probability and Measure, Willey, 1995.

[5] C. Borgs, J. T. Chayes, L. Lovász, V. T. Sós, and K. Vesztergombi, Convergent sequences of dense graphs. I. Subgraph frequencies, metric properties and testing, Adv. Math. 219 (2008), no. 6, 18011851.

[6] E. Bullmore and Sporns O., Complex brain networks: graph theoretical analysis of structural and functional systems, Nat. Rev. Neurosci. 10(3) (2009), 186-98.

[7] J.W. Cahn and J.E. Hilliard, Free energy of a nonuniform system. I. Interfacial free energy, J. Chem. Phys 28 (1958), 258.

[8] F. Dorfler and F. Bullo, Synchronization and transient stability in power networks and non-uniform Kuramoto oscillators, SICON 50 (2012), no. 3, 1616-1642.

[9] Paul Fife, Dynamics of internal layers and diffusive interfaces, SIAM, 1988.

[10] Taras Girnyk, Martin Hasler, and Yuri Maistrenko, Multistability of twisted states in non-locally coupled Kuramoto-type models, Chaos 22 (2012), 013114.

[11] Y. Kuramoto, Chemical Oscillations, Waves, and Turbulence, Springer, Berlin, 1984.

[12] Y. Kuramoto and D. Battogtokh, Coexistence of coherence and incoherence in nonlocally coupled phase oscillators, Nonlinear Phenomena in Complex Systems 5 (2002), 380-385.

[13] Yoshiki Kuramoto, Cooperative dynamics of oscillator community, Progress of Theor. Physics Supplement (1984), 223-240.

[14] R.D. Li and T. Erneux, Preferential instability in arrays of coupled lasers, Phys. Rev. A 46 (1992), $4252-4260$.

[15] László Lovász, Large networks and graph limits, AMS, Providence, RI, 2012.

[16] László Lovász and Balázs Szegedy, Limits of dense graph sequences, J. Combin. Theory Ser. B 96 (2006), no. 6, 933-957. MR 2274085 (2007m:05132)

[17] Georgi S. Medvedev, The nonlinear heat equation on dense graphs and graph limits, ArXiv e-prints, 1302.5804 (2013).

[18] Georgi S. Medvedev, The nonlinear heat equation on W-random graphs, Archive for Rational Mechanics and Analysis (2013), accepted. 
[19] R. Monasson, Diffusion, localization, and dispersion relations on 'small-world' lattices, Eur. Phys. J. B 12 (1999), 555-567.

[20] N.E.J. Newman and D.J. Watts, Renormalization group analysis of the small-world network model, Phys. Lett. A 263 (1999), 341-346.

[21] E. Niebur, H.G. Schuster, D.M. Kammen, and C. Koch, Oscillator phase coupling for different twodimensional network connectivities, Physical Review A 44 (1991), no. 10, 6895-6904.

[22] O.E. Omelchenko, M. Wolfrum, S. Yanchuk, Y. Maistrenko, and O. Sudakov, Stationary patterns of coherence and incoherence in two-dimensional arrays of non-locally-coupled phase oscillators, Physical Review E 85 (2012), 036210.

[23] Oleg Pikhurko, An analytic approach to stability, Discrete Math. 310 (2010), 2951-2964.

[24] Shinya Watanabe and Steven H. Strogatz, Constants of motion for superconducting Josephson arrays, Physica D: Nonlinear Phenomena 74 (1994), no. 34, 197 - 253.

[25] Duncan J. Watts and Steven H. Strogatz, Collective dynamics of small-world networks, Nature 393 (1998), 440-442.

[26] Daniel A. Wiley, Steven H. Strogatz, and Michelle Girvan, The size of the sync basin, Chaos 16 (2006), no. 1, 015103, 8. MR 2220552 (2007e:37016) 